\title{
Abscesos hepáticos piógenos de origen criptogenético
}

\author{
J. A. ÁLVAREZ PÉREZ, R. F. BALDONEDO CERNUDA, J. J. GONZÁLEZ \\ GONZÁLEZ*, L. SANZ ÁLVAREZ*, G. CARREÑO VILLARREAL**, \\ I. GARCÍA BEAR, J. I. JORGE BARREIRO \\ Servicio de Cirugía General. Hospital San Agustín. Avilés. *Hospital Central \\ Universitario. Universidad de Oviedo. **Hospital de Cabueñes. Gijón
}

PYOGENIC LIVER ABSCESSES OF CRYPTOGENIC ORIGIN

\section{RESUMEN}

Objetivo: Analizar y clarificar el comportamiento clínico y evolutivo de los pacientes con abscesos hepáticos piógenos de origen criptogenético y, además, observar si existen características diferenciales en relación a los abscesos de enfermos con etiopatogenia conocida.

Material y métodos: Estudio multicéntrico y retrospectivo de 2 grupos de pacientes diagnosticados de absceso hepático piógeno, durante un periodo de 13 años. Grupo 1: 34 casos, con abscesos de origen desconocido y grupo 2: 99 enfermos, con etiopatogenia conocida. Se analizaron y se compararon datos clínicos, biológicos y bacteriológicos, morfología y topografía lesional, tratamiento y evolución clínica.

Resultados: La duración de la sintomatología fue mayor en el Grupo 1 (9,4 $\pm 6,5$ versus $8,1 \pm 4,4$ días; $p=0,05)$. Aunque, también, en esta categoría de pacientes se observó menor frecuencia de ictericia (6 versus $26 \% ; \mathrm{p}=0,02)$ y menor nivel de bilirrubinemia $(0,9 \pm 0,9$ versus $2,6 \pm$ $4,4 \mathrm{mg} / \mathrm{dl} ; \mathrm{p}=0,05)$, la mayoría de los datos clínicos y biológicos fueron similares en ambos grupos. La proporción de pacientes con afectación del lóbulo izquierdo ( 26 versus $12 \% ; \mathrm{p}=0,04$ ) fue superior en el grupo 1 . La modalidad terapéutica efectuada en los dos grupos no fue significativamente diferente, como tampoco lo fue la estancia hospitalaria y las tasas de morbilidad y mortalidad.

Conclusiones: Los pacientes con abscesos hepáticos piógenos de origen criptogenético presentan escasas características clínicas específicas que faciliten su diagnóstico; además, su comportamiento evolutivo no fue significativamente diferente del hallado en enfermos con abscesos de etiopatogenia conocida.

PALABRAS CLAVE: Absceso hepático piógeno. Etiología. Clínica. Microbiología. Tratamiento. Evolución.

\section{ABSTRACT \\ Objective: To analyze and clarify the clinical behaviour and outco -} me of patients with pyogenic liver abscess of cryptogenic origin and, moreover, to observe if there are any differential characteristics in rela tion to those found in patients with a known pathogenesis.

Material and methods: Multicenter and retrospective study of two groups of patients diagnosed of pyogenic liver abscess, during a period of 13 years. Group 1: 34 cases with abscess of cryptogenic origin and Group 2: 99 patients with a known pathogenesis. Clinical, laboratory, and microbiologic data, morphology and topography of the lesions, tre atment and outcome were assessed and compared in both groups.

Results: The duration of symptomatology was higher in Group $1(9.4$ \pm 6.5 vs $8.1 \pm 4.4$ days; $p=0.05)$. Although, in this category of patients, it was also observed a lower frequency of jaundice (6 vs 26\%; $p=0.02$ ) and lower level of bilirubinemia $(0.9 \pm 0.9 \mathrm{vs} 2.6 \pm 4.4 \mathrm{mg} / \mathrm{dl} ; p=0.05)$, the majority of clinical and laboratory data were similar in both groups. The rate of patients with abscesses on the left lobe $(26 \%$ vs $12 \%$; $p=$ $0.04)$ was higher in Group 1. The therapeutic modality carried out in the two groups was not significantly different, neither were hospital stay, and morbidity and mortality rates.

Conclusions: The patients with pyogenic liver abscesses of cryptoge nic origin present few specific clinical characteristics which facilitate the diagnosis. Besides, their outcome was no significantly different from that found in patients with abscesses of known pathogenesis.

KEY WORDS: Pyogenic liver abscess. Etiology. Clinical features. Microbiology. Treatment. Outcome.

Álvarez Pérez JA, Baldonedo Cernuda RF, González González JJ, Sanz Álvarez L, Carreño Villarreal G, García Bear I, Jorge Barreiro JI. Abscesos hepáticos piógenos de origen criptogenético. An Med Interna (Madrid) 2001; 18: 473-478.

\section{INTRODUCCIÓN}

El absceso hepático piógeno es una enfermedad que, históricamente, ha sido considerada de diagnóstico dificultoso y manejo terapéutico complicado, asociándose a elevada mortalidad (1). En las últimas décadas, a pesar de los avances antibioticoterápicos y de la introducción de técnicas radiológicas, como la ecografía y la TAC, que permitieron el desarrollo de técnicas conservadoras, la tasa de mortalidad en pacientes quirúrgicos y en los tratados mediante drenaje percutáneo, aún se sitúa entre $12-26$ y $2-15 \%$, respectivamente (2-5). Estos no tan favorables resultados se atribuyen al incremento en el número de pacientes con neoplasias, patología biliar compleja, edad avanzada y estados de inmunosupresión postquimioterapia.

Trabajo aceptado: 14 de marzo de 2001

Correspondencia: Jose Antonio Álvarez Pérez. Avenida de Galicia, 46-3º. 33005 Oviedo. 
El desconocimiento del mecanismo etiopatogénico de estos procesos supurativos es un hecho que se sigue constatando en series recientes (6-8). El origen criptogenético, como factor de riesgo en este tipo de enfermos, es una circunstancia poco comentada en la literatura $(9,10)$, siendo prácticamente inexistentes los artículos que, de una manera específica, examinan los patrones de presentación clínica y el curso evolutivo de los casos con abscesos de causa no aclarada.

El objetivo de este estudio es analizar y clarificar el comportamiento clínico y, sobre todo, evolutivo de los pacientes con abscesos hepáticos piógenos de origen criptogenético, y además, observar si existen características diferenciales en relación a los abscesos de enfermos con etiopatogenia conocida.

\section{MATERIAL Y MÉTODOS}

En cinco hospitales de la Comunidad Autónoma Asturiana y durante un periodo comprendido entre enero de 1985 y diciembre de 1997, 133 pacientes fueron diagnosticados de absceso hepático piógeno único o múltiple, siendo éstos revisados retrospectivamente. Dichos enfermos, fueron divididos en dos grupos: 34 casos en los que el origen del absceso era desconocido (grupo 1) y 99 enfermos, con etiopatogenia conocida (grupo 2).

\section{CRITERIOS DE INCLUSIÓN}

Todos los pacientes incluidos en el estudio cumplían, al menos, alguno de los criterios que detallamos a continuación:

- Hallazgo de un absceso hepático piógeno único o múltiple en una intervención quirúrgica.

-Demostración de una o varias masas intrahepáticas, de características líquidas mediante ecografía y/o TAC, con posterior drenaje de pus.

-Visualización de una o varias cavidades líquidas intrahepáticas, por medio de ecografía y/o TAC, junto con una fundada sospecha clínica, basada ésta en datos clínicos, biológicos y bacteriológicos.

\section{CRITERIOS DE EXCLUSIÓN}

Fueron excluidos del estudio, los pacientes con abscesos hepáticos de origen amebiano, micótico, los secundarios a hidatidosis infectada y aquéllos diagnosticados en exámenes necrópsicos.

\section{PROTOCOLO DE ESTUDIO}

Los aspectos clínicos revisados y comparados en ambos grupos fueron: edad, sexo, enfermedades asociadas, manifestaciones clínicas, datos de laboratorio, morfología y topografía lesional, hallazgos bacteriológicos, método de tratamiento y evolución clínica.

El origen criptogenético de los abscesos fue considerado cuando éstos aparecieron en pacientes en los que ni en los estudios diagnósticos ni en la intervención quirúrgica pudo identificarse ningún foco infeccioso causal. Los diferentes mecanismos etiopatogénicos de los pacientes del grupo 2 fue- ron los siguientes: por contigüidad, 39 casos; biliar, 26; portal 17; postcirugía hepática reciente, 7; arteria hepática, 5 y traumático, 5. Se analizó la sintomatología clínica y la duración de la misma. Los datos de laboratorio evaluados fueron: recuento leucocitario, hemoglobina, aspartato aminotransferasa (AST), fosfatasa alcalina, bilirrubina total, urea, creatinina, albúmina, tiempo de protrombina y tiempo de tromboplastina parcial activada. También se estudió el índice de gravedad pretratamiento en los pacientes de ambos grupos, mediante la escala APACHE II (11). Las técnicas radiológicas utilizadas fueron la Rx. de tórax, Rx. simple de abdomen, ecografía y TAC; mediante las mismas, se definió la morfología y la topografía lesional (número, tamaño y distribución lobar). En los estudios bacteriológicos, los gérmenes obtenidos de cultivos de pus y hemocultivos fueron aislados e identificados mediante técnicas rutinarias estándar aeróbicas y anaeróbicas.

Todos los pacientes fueron tratados con antibióticos de amplio espectro, por vía parenteral, generalmente tras la obtención de muestras para hemocultivos, modificándose, posteriormente, según los resultados microbiológicos. La antibioticoterapia, como único tratamiento, se indicó, en general, en lesiones múltiples o únicas de pequeño tamaño. La indicación de drenaje quirúrgico fue determinada por la localización, número y tamaño del absceso, por la existencia de patología abdominal asociada y por la situación clínica del enfermo; así, en pacientes que presentaron litiasis biliar complicada, absceso roto con peritonitis, dificultades técnicas en la práctica de drenaje percutáneo o bien ausencia de respuesta tras la realización de éste, fueron seleccionados para drenaje operatorio. Cuando se indicó un drenaje percutáneo, éste se realizó mediante ecografía o TAC. Después de la aspiración diagnóstica, se colocó un catéter de doble luz, según la técnica modificada de Seldinger. Tras el drenaje, el control evolutivo fue realizado con ecografía y/o TAC. Si la situación clínica del paciente mejoraba, tras el tratamiento médico o tras el drenaje percutáneo del absceso, la terapia antibiótica se prolongaba durante 4-6 semanas. Se realizó hepatectomía atípica cuando el absceso producía destrucción importante del parénquima hepático.

La evolución clínica fue estudiada en relación al tiempo de hospitalización, y a la tasa de curso clínico complicado, curso clínico complicado relacionado y mortalidad hospitalaria. Después del tratamiento, el curso clínico fue considerado complicado (CCC), cuando el paciente presentó cualquier tipo de complicación, y complicado relacionado (CCCR) si: a) el paciente permaneció séptico, b) se comprobó aumento de la lesión, c) se constató recidiva del absceso o aparecieron nuevas localizaciones, d) el procedimiento de drenaje no fue efectivo, a pesar del uso de drenajes de calibre progresivamente grande, o e) si, adicionalmente, fue necesaria una intervención quirúrgica para la resolución del absceso o de patología abdominal asociada. La mortalidad fue definida como la habida hasta 30 días después del comienzo del tratamiento, o bien, antes del alta hospitalaria.

\section{ANÁLISIS ESTADÍSTICO}

Los datos recogidos fueron analizados mediante el paquete bioestadístico SPSS para Windows, versión 8.0 (SPSS Inc). Las variables continuas fueron expresadas en función de su media \pm desviación estándar y las variables categóricas como 
porcentajes. Para la comparación de medias se utilizaron las pruebas de la t de Student, o de la U de Mann-Whitney en el caso de que la distribución fuese no paramétrica, y para comparar proporciones, el test de la $\chi^{2}$ o el test extacto de Fisher, cuando fue preciso. Se consideró significación estadística cuando $\mathrm{p}<0,05$.

\section{RESULTADOS}

Entre los 133 pacientes, el origen predominante de los abscesos fue el tracto biliar, 57 casos (43\%). Del total de enfermos que componen la serie, 34 casos $(26 \%)$ corresponden al grupo 1 (abscesos criptogenéticos) y 99 casos (74\%) al grupo 2 (abscesos con etiopatogenia conocida). En la primera mitad del estudio, el origen criptogenético de los abscesos fue identificado en 12 de 47 enfermos (25\%) y en la segunda, en 22 de $86(26 \%)$, respectivamente.

La edad media del grupo 1 y 2 fue $57 \pm 20,2$ y $61 \pm 17,5$ años, mientras que la relación hombre/mujer fue de 17:17 y $65: 34$, respectivamente. Se detectó alguna enfermedad asociada en 18 casos (53\%) del grupo estudio y en 64 casos $(65 \%)$ del grupo comparado. Entre ellas, la presencia de diabetes, en ambas categorías de pacientes, fue objetivada en $6(18 \%)$ y en 11 casos $(11 \%)(\mathrm{p}=0,32)$; mientras que la existencia de un proceso maligno asociado fue observado en $5(15 \%)$ y en 29 enfermos $(29 \%)$, respectivamente $(\mathrm{p}=0,09)$.

La duración de la sintomatología en los dos grupos fue de $9,4 \pm 6,5$ y $8,1 \pm 4,4$ días, respectivamente $(p=0,05)$. Las principales manifestaciones clínicas se reflejan en la tabla I. En ella, destaca que la fiebre y el dolor abdominal fueron los hallazgos más habituales, y que la ictericia se objetivó con menor frecuencia en el grupo 1. Los datos biológicos (Tabla II) muestran que también en este grupo, y en sintonía con los datos clínicos, se detectó un menor nivel de bilirrubinemia media. El índice APACHE II en el grupo estudio $(6 \pm 8,1)$ fue menor que el hallado en el grupo comparado $(8 \pm 4,4)(\mathrm{p}=$ $0,06)$.

La morfología y la topografía de las lesiones se reseñan en la tabla III. En los dos grupos, la mayoría de los abscesos fueron únicos y de localización preferente en el lóbulo derecho,

\section{TABLA I}

\begin{tabular}{|c|c|c|}
\hline \multicolumn{3}{|c|}{ PRESENTACIÓ N CLÍNICA } \\
\hline Datos clínicos & $\begin{array}{c}\text { Grupo } 1 \\
\mathrm{~N}(\%)\end{array}$ & $\begin{array}{c}\text { Grupo } 2 \\
\mathrm{~N}(\%)\end{array}$ \\
\hline $\begin{array}{l}\text { Fiebre y escalofríos } \\
\text { Dolor abdominal } \\
\text { Adelgazamiento } \\
\text { Vómitos } \\
\text { Ictericia } \\
\text { Distrés respiratorio } \\
\text { CID } \\
\text { Shock } \\
\text { Hepatomegalia } \\
\text { Derrame pleural } \\
\text { Rotura }\end{array}$ & $\begin{array}{c}33(97) \\
23(68) \\
13(38) \\
11(32) \\
2(6)^{*} \\
1(3) \\
- \\
1(3) \\
11(32) \\
9(26) \\
2(6)\end{array}$ & $\begin{array}{c}89(90) \\
69(70) \\
43(43) \\
27(27) \\
26(26) \\
5(5) \\
4(4) \\
7(7) \\
22(22) \\
34(34) \\
5(5)\end{array}$ \\
\hline
\end{tabular}

CID: Coagulación intravascular diseminada; $\mathrm{N}=$ número de pacientes. $* p=0,02$.
TABLA II

\begin{tabular}{lcc}
\multicolumn{3}{c}{ TABLA II } \\
\hline \multicolumn{3}{c}{ DATOS DE LABO RATO RIO } \\
Parámetro & media \pm DE & media \pm DE \\
\hline Recuento leucocitario (/mm ${ }^{3}$ ) & $14,176 \pm 7,155$ & $15.648 \pm 7.899$ \\
Hemoglobina (g/dl) & $11,9 \pm 2,1$ & $11,4 \pm 2,1$ \\
Fosfatasa alcalina (UI/l) & $306 \pm 235$ & $657 \pm 971$ \\
Bilirrubina total (mg/dl) & $0,9 \pm 0,9 *$ & $2,6 \pm 4,4$ \\
AST (UI/l) & $57 \pm 41$ & $76 \pm 121$ \\
Urea (mg/dl) & $46 \pm 37$ & $47 \pm 31$ \\
Creatinina (mg/dl) & $1,1 \pm 0,4$ & $1,2 \pm 0,6$ \\
Albúmina (g/dl) & $3,0 \pm 0,6$ & $3,1 \pm 0,6$ \\
T. de protrombina (seg) & $13 \pm 1,5$ & $15 \pm 7,1$ \\
T. de tromboplastina parcial & & \\
$\quad$ activada (seg) & $29 \pm 4,1$ & $31 \pm 7,6$ \\
\hline
\end{tabular}

DE: desviación estándar; AST : aspartato aminotransaminasa. ${ }^{*} p=0,05$.

TABLA III

MORFOLOGÍA Y TOPOGRAFÍA DE LOS ABSCESO S

\begin{tabular}{lcc}
\hline Característica & $\begin{array}{c}\text { Grupo } 1 \\
\text { N (\%) }\end{array}$ & $\begin{array}{c}\text { Grupo 2 } \\
\text { N (\%) }\end{array}$ \\
\hline Localización & $24(71)$ & $71(72)$ \\
$\quad$ Lóbulo derecho & $9(26)^{*}$ & $12(12)$ \\
$\quad$ Lóbulo izquierdo & $1(3)^{* *}$ & $16(16)$ \\
$\quad$ Bilobar & & \\
Número & $27(79)$ & $70(71)$ \\
$\quad$ Único & $7(21)$ & $29(29)$ \\
Múltiple & $7,6 \pm 3,1$ & $6,6 \pm 3,0$ \\
\hline
\end{tabular}

avalores expresados en media \pm desviación estándar; $\mathrm{N}=$ número de pacientes. $* p=0,04 ; * * p=0,03$.

no obstante, el asiento en el lóbulo hepático izquierdo fue más común en el grupo 1, mientras que la afectación bilobar fue más frecuente en el grupo 2. La visualización radiológica de gas en el absceso fue objetivada en 5 casos (15\%) del grupo estudio y en 24 (24\%) del grupo comparado $(\mathrm{p}=0,24)$.

Entre los pacientes del grupo 1, el estudio bacteriológico de los hemocultivos mostró positividad en 9 enfermos (26\%) y cultivos estériles en 14 (42\%), mientras que en 11 casos (32\%) no se realizaron. En 7 pacientes se aislaron agentes aerobios; en cambio, los anaerobios sólo se identificaron en 2 casos. El microorganismo detectado con mayor frecuencia fue Streptococcus milleri, seguido de E. coli. Entre los enfermos del grupo 2, los hemocultivos fueron positivos en 42 (43\%), estériles en $25(25 \%)$, y no se realizaron en 32 (32\%). Entre los 42 pacientes de este grupo con hemocultivos positivos se aislaron bacterias aerobias en 38, mientras que los anaerobios fueron recogidos en sólo 6 ocasiones. En este grupo, el gérmen más frecuente fue E. coli, seguido de Streptococcus milleri. El estudio bacteriológico del pus de los abscesos del grupo 1 reveló cultivos positivos en 21 pacientes (62\%) y estériles en 4 (12\%), mientras que en 9 casos (26\%) no se realizaron. En los 21 enfermos con positividad en los cultivos de 
pus, se aislaron aerobios en 17 y anaerobios en 6. También el agente etiológico aislado con mayor frecuencia fue Strepto coccus milleri, seguido de E. coli. En el grupo 2, los cultivos de pus fueron positivos en 63 (64\%), estériles en 10 (10\%), y no se realizaron en 26 (26\%). Entre los 63 enfermos con cultivo de exudado positivo, se detectaron aerobios en 58 ocasiones, y anaerobios en 19. El microorganismo más común fue $\mathrm{E}$. coli, seguido estrechamente del Streptococcus milleri. En el análisis comparativo de estos hallazgos, sólo es de resaltar que la mayor tasa de hemocultivos estériles en los pacientes del grupo 1 casi alcanzó la significación estadística $(p=0,07)$, no existiendo ninguna diferencia significativa, entre los dos grupos, en cuanto al tipo de germen causal aislado. Entre los pacientes con cultivos de pus positivo, la infección fue polimicrobiana en 6 casos (29\%) del grupo 1, y en $25(40 \%)$ del grupo $2(\mathrm{p}=0,36)$.

Los métodos de tratamiento realizados en los grupos 1 y 2 fueron, antibioticoterapia aislada en $5(15 \%)$ y $20(20 \%)(\mathrm{p}=$ $0,48)$; drenaje percutáneo en $19(56 \%)$ y $44(44 \%)(\mathrm{p}=0,25)$, y drenaje quirúrgico en $10(29 \%)$ y 35 enfermos $(36 \%)$ (p = 0,52 ), respectivamente. Entre los operados, se realizó hepatectomía en 3 pacientes, correspondientes todos ellos al grupo 2. En relación a la evolución clínica, la estancia media hospitalaria en el grupo estudio fue de $29 \pm 14,3$ días y en el grupo comparado, $37 \pm 19,2$ días $(p=0,10)$. La tasa global en la serie de CCC, CCCR y mortalidad fue de 36,1, 21,8 y 14,3\%, respectivamente. Las causas de todos los fallecimientos fueron absceso-relacionadas. Los porcentajes de CCC y CCCR y mortalidad de ambos grupos no fueron significativamente diferentes (Fig. 1). La tasa de mortalidad según los diferentes métodos terapéuticos utilizados se refleja en la tabla IV; en la misma, se observa que el drenaje percutáneo y la terapia antibiótica aislada conllevaron una mortalidad similar, sin embargo, cuando los pacientes fueron tratados mediante drenaje quirúrgico, ésta fue superior en el grupo 2, aunque de modo no significativo.

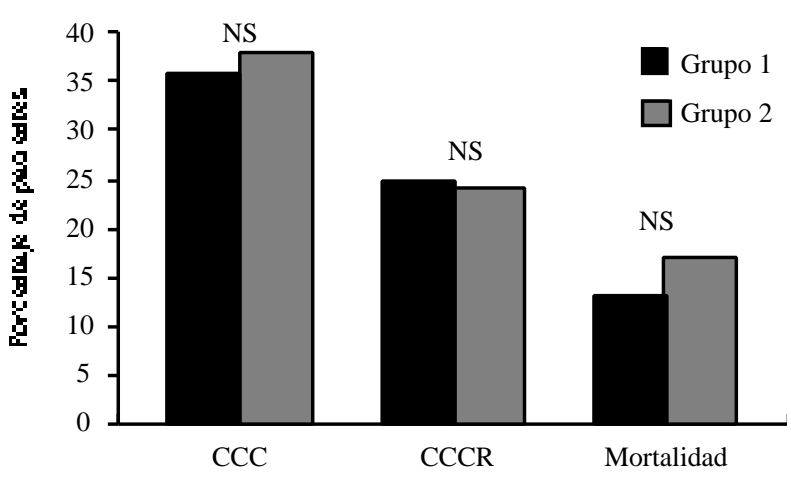

Fig. 1. Evolución clínica postratamiento, según la existencia de curso clínico complicado (CCC), curso clínico complicado relacionado (CCCR) y mortalidad; NS: no significativo.

\section{DISCUSIÓN}

El origen de los abscesos hepáticos piógenos, en una proporción variable de pacientes, es desconocido. El razonamiento aducido para explicar la etiopatogenia criptogenética es diverso. Así, se ha relacionado con infecciones no detectadas
TABLA IV

M ORTALIDAD SEGÚN EL TIPO DE TRATAM IENTO APLICADO*

\begin{tabular}{lcc}
\hline & $\begin{array}{c}\text { Grupo 1 } \\
\text { N/N } \\
\text { total (\%) }\end{array}$ & $\begin{array}{c}\text { Grupo 2 } \\
\text { N/N } \\
\text { total (\%) }\end{array}$ \\
M étodo & $1 / 5(20)$ & $4 / 20(20)$ \\
\hline Antibioticoterapia aislada & & \\
Drenaje percutáneo & $3 / 19(16)$ & $5 / 44(11)$ \\
$\quad$ Aspiración & $1 / 4$ & $1 / 8$ \\
Catéter-drenaje & $2 / 15$ & $4 / 36$ \\
Procedimientos quirúrgicos & $0 / 10(0)$ & $6 / 35(17)$ \\
Drenaje & - & $6 / 32$ \\
Hepatectomía & - & $0 / 3$ \\
\hline
\end{tabular}

* En ninguna de las modalidades terapéuticas se constataron diferencias significativas entre los grupos comparados; N: número de pacientes.

o ya resueltas en el territorio portal $(2,12)$, de ahí que algunos propugnen, en estos casos, una evaluación completa del tracto gastrointestinal, ya sea de una manera sistemática (13) o selectiva (5). Otros refieren la existencia de una disfunción subyacente del sistema retículoendotelial (14). En suma, que aunque todas estas posibilidades son atractivas desde el punto de vista patogénico, son aún meras hipótesis. Globalmente, en nuestra serie, el origen más común de los abscesos se localizó en el árbol biliar, en consonancia con lo mencionado en la literatura $(4,6,7)$. Por su parte, el origen criptogenético tuvo especial importancia, como lo demuestra el hecho de ser la segunda forma etiopatogénica en frecuencia (26\%), manteniéndose constante a lo largo de todo el estudio; dicho porcentaje fue similar al descrito por otros autores $(4,8)$; sin embargo, en la actualidad aún se publican series donde el origen desconocido de los abscesos es más elevado e incluso predominante $(5,15-17)$.

Algunos investigadores (5) han comunicado que los pacientes con abscesos criptogenéticos son más jóvenes que los de otras formas etiológicas, circunstancia no confirmada en nuestra serie, donde la edad media del grupo estudio fue similar a la del grupo comparado, al igual que lo referido en otras revisiones $(6,18)$. El predominio sobre el sexo masculino, frecuente en los pacientes con abscesos piógenos con $(18,19)$ o sin patogenia conocida $(5,14)$, no fue objetivado en el grupo con abscesos criptogenéticos de nuestro estudio, siendo la afectación por sexos la misma. Diversos artículos $(10,20)$ han reflejado una mayor frecuencia de diabetes mellitus en los enfermos con abscesos criptogenéticos; este hallazgo no fue confirmado por nosotros, como, también, se cita en una trabajo reciente (16).

Se ha referido que la duración de la sintomatología en los enfermos con abscesos piógenos varía según el tipo de enfermedad subyacente, de tal manera que aquellos con patología biliar o diseminación por vía arterial tienen una periodo sintomático más corto que los de patogenia portal o criptogenética (5); esta situación fue vista en nuestro trabajo, donde la mayor duración de los síntomas en el grupo estudio alcanzó casi la significación estadística; no obstante, hay autores (14) que mencionan resultados discordantes. La presentación clínica no ayudó a distinguir específicamente a los abscesos piógenos 
de etiología desconocida, y no difirió sustancialmente de la hallada en pacientes con abscesos de causa conocida. La fiebre con escalofríos y el dolor abdominal fueron los hallazgos clínicos que sobresalieron en ambos grupos. En los enfermos con abscesos criptogenéticos es habitual objetivar niveles normales o mínimas elevaciones de bilirrubina o de fosfatasa alcalina (5); los resultados de nuestra serie estuvieron en esa línea; así, entre los pacientes con este tipo de abscesos se observó menor nivel de bilirrubinemia y menor frecuencia de ictericia, que en aquellos con lesiones de etiología conocida.

El estudio morfológico y topográfico de los abscesos puso de manifiesto que, en los dos grupos, la mayoría de los mismos fueron únicos y de localización preferente en el lóbulo hepático derecho, como ya ha sido descrito $(5,16,21)$. Además, se observó, sin una razón aparentemente clara, que la afectación del lóbulo izquierdo fue más común en el grupo criptogenético.

El análisis bacteriológico mostró cierta tendencia a presentar mayor tasa de hemocultivos estériles en el grupo estudio, hallazgo ya subrayado por Seeto and Rockey (5). Aunque el tipo de microorganismo aislado, tanto en los hemocultivos como en los cultivos de pus, no fue significativamente diferente en las dos categorías de enfermos, el agente patógeno más comúnmente identificado en los abscesos criptogenéticos fue Streptococcus milleri, destacando la importancia que, en los últimos años, se ha atribuído a este agente en la génesis de los abscesos piógenos $(8,18,22,23)$. Este microorganismo, también tuvo especial prevalencia en los enfermos con abscesos de patogenia conocida, sin embargo, los Gram-negativos fueron los gérmenes aislados con mayor frecuencia, entre ellos, la bacteria más identificada en este grupo fue $E$. coli, en sintonía con diversas series $(4,6,18,19,24)$. También se constató una baja tasa de anaerobios en los cultivos de exudado. Otra característica bacteriológica citada en la literatura (5), es la existencia de un menor porcentaje de infección polimicrobiana en los abscesos criptogenéticos; no obstante, dicho aspecto no fue corroborado estadísticamente en nuestro trabajo.

\section{Bibliografía}

1. Ochsner A, DeBakey M, Murray S. Pyogenic abscess of the liver. An analysis of 47 cases with review of the literature. Am J Surg 1938; 40: 292-319.

2. Miedema BW, Dinnen P. The diagnosis and treatment of pyogenic liver abscesses. Ann Surg 1984; 200: 328-335.

3. Greenstein AJ, Lowenthal D, Hammer GS, Schaffner F, Aufses AH, Jr. Continuing changing patterns of disease in pyogenic liver abscess: A study of 38 patients. Am J Gastroenterol 1984; 79: 217-226.

4. Branum GD, Tyson GS, Branum MA, Meyers WC: Hepatic abscess. Changes in etiology, diagnosis, and management. Ann Surg 1990; 212: 655-662.

5. Seeto RK, Rockey DC. Pyogenic liver abscess: Changes in etiology, management, and outcome. Medicine 1996; 75: 99-113.

6. Huang CJ, Pitt HA, Lipsett PA, Osterman FA Jr, Lillemoe KD, Cameron JL et al. Pyogenic hepatic abscess. Changing trends over 42 years. Ann Surg 1996; 223: 600-609.

7. Lee KT, Sheen PC, Chen JS, Ker CG. Pyogenic liver abscess: Multivariate analysis of risk factors. World J Surg 1991; 15: 372-377.

8. Corredoira Sánchez JC, Casariego Vales E, Ibáñez Alonso MD, Rigueiro Veloso MT, Coira Nieto A, Alonso García P et al. Absceso hepático
El moderno enfoque terapéutico en los pacientes con abscesos hepáticos piógenos apunta hacia una preponderancia de los métodos percutáneos $(6,8,25-32)$. Esta actitud se confirmó en nuestro estudio; así, en la primera parte del mismo (19851991), 21 de 47 pacientes (45\%) con esta enfermedad fueron inicialmente tratados quirúrgicamente, sin embargo, en los últimos 6 años, sólo en 24 de 86 pacientes (28\%) se empleó la opción quirúrgica. Globalmente, y aunque de forma no significativa, también se apreció cierta tendencia a realizar una terapéutica menos agresiva en los pacientes del grupo criptogenético, como se refleja en el hecho de haber menos abscesos drenados quirúrgicamente y más por métodos percutáneos, al igual que se menciona en otros artículos (5).

En relación al curso clínico de los pacientes con abscesos hepáticos piógenos de origen criptogenético, algunos (14) señalan una elevada tasa de morbilidad absceso-relacionada en estos enfermos, mientras que otros describen un curso evolutivo más favorable que cuando existe una etiología conocida $(3,5)$. También esta forma patogénica ha sido analizada, específicamente, como factor pronóstico de mortalidad, sin haber podido confirmar su utilidad como valor predictivo $(9,33)$. Por último, hay autores (10) que refieren la misma mortalidad en estos pacientes que en aquéllos con abscesos de causa aclarada. Nuestros resultados están en esa línea, de tal modo que, tanto la estancia hospitalaria como las tasas de morbilidad y de mortalidad, no fueron significativamente diferentes en ambos tipos de enfermos. El análisis de la mortalidad, según el tipo de tratamiento aplicado, demostró que no hubo diferencias estadísticas entre los dos grupos, no obstante, es de destacar que, entre los casos con abscesos drenados quirúrgicamente, no existió mortalidad en los de origen criptogenético.

Tras los resultados del estudio, se puede concluir con que los pacientes con abscesos hepáticos piógenos de origen criptogenético presentan escasas características clínicas específicas que faciliten su diagnóstico; además, su comportamiento evolutivo fue similar al hallado en enfermos con abscesos de etiopatogenia conocida. piógeno: cambios en la etiología, diagnóstico y tratamiento a lo largo de 18 años. Rev Clin Esp 1999; 199: 705-710.

9. Bowers ED, Robison DJ, Doberneck RC. Pyogenic liver abscess. World J Surg 1990; 14: 128-132.

10. Chou FF, Sheen-Chen SM, Chen YS, Chen MC, Chen FC, Tai DI. Prognostic factors for pyogenic abscess of the liver. J Am Coll Surg 1994; 179: 727-732.

11. Knaus WA, Draper EA, Wagner DP, Aimmerman JE. APACHE II: a severity of disease classification. Crit Care Med 1985; 13: 818-829.

12. Wallack MK, Brown AS, Austrian R, Fitts WT Jr. Pyogenic liver abscess secondary to asymptomatic sigmoid diverticulitis. Ann Surg 1976; 184: 241-243.

13. Cohen JL, Martín MS, Rossi RL, Schoetz DJ Jr. Liver abscess: The need for complete gastrointestinal evaluation. Arch Surg 1989; 124: 561-4.

14. Minuk GY, Nicole LE, Sherman T. Cryptogenic abscess of the liver. Evidence of underlying reticuloendothelial cell dysfunction. Arch Surg 1987; 122: 906-908.

15. Corbella X, Vadillo M, Torras J, Pujol M, Rafecas A, Gudiol F. Presentación, diagnóstico y tratamiento del absceso hepático piógeno: análisis de una serie de 63 casos. Enf Infecc Microbiol Clin 1995; 13: 80-84. 
16. Chu KM, Fan ST, Lai ECS, Lo CM, Wong J. Pyogenic liver abscess: An audit of experience over the past decade. Arch Surg 1996; 131: 148-152.

17. Ramos A, Gazapo T, Murillas J, Martin H, Mendaza P, Cuervas-Mons V. Absceso hepático piógeno. Gastroenterol Hepatol 1996; 19: 292-296.

18. Barrio J, Cosme A, Ojeda E, Garmendia G, Castiella A, Bujanda L et al. Abscesos hepáticos piógenos de origen bacteriano. Estudio de una serie de 45 casos. Rev Esp Enf Digest 2000; 92: 233-235.

19. McDonald MI, Corey GR, Gallis HA, Durack DT. Single and multiple pyogenic liver abscess. Medicine 1984; 63: 291-302.

20. Yang CC, Chen CY, Lin XZ, Chang TT, Shin JS, Lin CY. Pyogenic liver abscess in Taiwan: emphasis on gas-forming liver abscess in diabetics. Am J Gastroenterol 1993; 88: 1911-1915.

21. Chou FF, Sheen-Chen SM, Chen YS, Chen MC. Single and multiple pyogenic liver abscesses: Clinical course, etiology, and results of treatment. World J Surg 1997; 21: 384-389.

22. Corredoira J, Casariego E, Moreno C, Villanueva L, López MJ, Varela J et al. Prospective study of Streptococcus milleri hepatic abscess. Eur J Clin Microbiol Infect Dis 1998; 17: 556-560.

23. Martín-Pérez E, Galván A, Larrañaga E, Figueroa JM, Serrano PA. Absceso hepático piogénico como complicación de la enfermedad de Crohn. An Med Interna (Madrid) 2000; 17: 657-659.

24. Blanco Quintana F, Novella Arribas B, Sánchez Molini P, Sanz Sanz J. Estudio descriptivo de 39 casos de abscesos hepáticos de origen piógeno y amebiano. An Med Interna (Madrid) 1995; 12: 477-484.
25. Rintoul R, O’ Riordain MG, Laurenson IF, Crosbie JL, Allan PL, Garden OJ. Changing management of pyogenic liver abscess. Br J Surg 1996; 83: 1215-1218.

26. Lambiase RE, Deyoe L, Cronan JJ, Dorfman GS. Percutaneous drainage of 335 consecutive abscesses: results of primary drainage with 1-year follow-up. Radiology 1992; 184: 167-179.

27. Giorgio A, Tarantino L, Mariniello N, Francica G, Scala E, Amoroso P et al. Pyogenic liver abscesses: 13 years of experience in percutaneous needle aspiration with US guidance. Radiology; 1995; 195: 122-124.

28. Stain SC, Yellin AE, Donovan AJ, Brien HW. Pyogenic liver abscess: Modern treatment. Arch Surg 1991; 126: 991-996.

29. Wong KP. Percutaneous drainage of pyogenic liver abscesses. World J Surg 1990; 14: 492-497.

30. Hashimoto L, Hermann R, Grundfest-Broniatowski S. Pyogenic hepatic abscess: Results of current management. Am Surg 1995; 61: 407-411.

31. De Miguel J, Díaz F, Vidales L, Miguelez JL, Collazos J, Marco A. Abscesos hepáticos piógenos. Estudio de 20 pacientes tratados mediante drenaje percutáneo. An Med Interna (Madrid) 1994; 11: 27-30.

32. Rajak CL, Gupta S, Jain S, Chawla Y, Gulati M, Suri S. Percutaneous treatment of liver abscesses: needle aspiration versus catheter drainage. Am J Roentgenol 1998; 170: 1035-1039.

33. Álvarez JA, González JJ, Baldonedo RF, Sanz L, Garreño G, Junco A et al. Clinical course, treatment, and multivariate analysis of risk factors for pyogenic liver abscess. Am J Surg (en prensa). 